\title{
TOTALLY COMMUTATIVE SEMIGROUPS
}

\author{
JÓZEF DUDEK and ANDRZEJ KISIELEWICZ
}

(Received 4 April 1989; revised 23 February 1990)

Communicated by P. G. Trotter

\begin{abstract}
A semigroup is totally commutative if each of its essentially binary polynomials is commutative, or equivalently, if in every polynomial (word) every two essential variables commute. In the present paper we describe all varieties (equational classes) of totally commutative semigroups, lattices of subvarieties for any variety, and their free spectra.

1991 Mathematics subject classification (Amer. Math. Soc.) 20 M 07, 20 M 05, 08 B 15; Sec-



In $[2,7,8]$ all varieties of idempotent semigroups are described. A semigroup $\langle S, x y\rangle$ is idempotent, if $x y$ is idempotent, that is the identity $x^{2}=x$ holds. Any idempotent semigroup is totally idempotent in the sense that each of its binary polynomials is idempotent, as well. In this paper we consider from this point of view the commutative law

$$
f(x, y)=f(y, x) .
$$

In contrast with idempotency, this identity is interesting only for operations depending on both the variables (that is, essentially binary), since otherwise (1) fails to hold, unless $f(x, y)$ is a constant operation.

Definition. A semigroup $S$ is totally commutative (in short, a TC-semigroup) if for every essentially binary polynomial $f(x, y)$ of $\mathbb{S}$ the identity (1) holds.

Boolean groups and semilattices are important and well-known examples of TC-semigroups. Left and right zero semigroups are examples of improper TC-semigroups (see [17] and Section 2 below).

(C) 1991 Australian Mathematical Society 0263-6115/91 $\$ A 2.00+0.00$ 
We show that the class of TC-semigroups is the union of four equational classes. There are $\aleph_{0}$ distinct varieties of TC-semigroups and all the varieties are finitely based. For each variety of TC-semigroups normal forms for words are found, solutions for word problems are explicitly shown and the lattices of subvarieties are completely described. For these kind of problems considered for various classes of semigroups see $[10,11,12,15]$ and [19] for further references. As in [9], we also describe $p_{n}$-sequences of all the varieties, and in consequence, cardinalities of free semigroups (free spectra). From this point of view the class of TC-semigroups is especially interesting, providing the most natural examples of free spectra with the slowest growth (for this topic see also $[5,13,16])$. Finally, in the last section, a certain contribution to the topic of hyperidentities is given (see $[1,3,4,18]$ ).

For the terminology used in this paper the reader is referred to [19].

\section{General remarks}

A variable $x_{i}$ in a polynomial $p=p\left(x_{1}, \ldots, x_{n}\right)$ is called essential if the value of $p$ does really depend on $x_{i}$; for a more precise definition see [13]).

Proposition 1.1. A semigroup $\mathbb{S}$ is a TC-semigroup if and only if every polynomial $p=p\left(x_{1}, \ldots, x_{n}\right)$ over $\mathbb{S}$ is symmetric in all essential variables.

Proof. The "if" part is trivial. For the "only if" part let $\mathrm{S}=\langle X, x y\rangle$ and note that either $x y$ is not essentially binary (then each polynomial in $\$$ pends on at most one variable and the result is trivial) or $x y$ depends on both the variables, and in consequence $\mathbb{S}$ is commutative. Let $p=x_{1}^{a_{1}} x_{2}^{a_{2}} \cdots x_{n}^{a_{n}}$, be an arbitrary polynomial of $\mathbb{S}$ and assume that $p$ depends on each of the variables $x_{i}$. It follows that $x_{i}^{a_{i}} x_{j}^{a_{j}}$ is an essentially binary operation, and by the assumption, $x_{i}^{a_{i}} x_{j}^{a_{j}}=x_{j}^{a_{i}} x_{i}^{a_{j}}$. Hence $p=x_{1 \pi}^{a_{1}} x_{2 \pi}^{a_{2}} \cdots x_{n \pi}^{a_{n}}$ for any permutation $\pi$ of indices $1,2, \ldots, n$, as required.

We note that the equivalence in Proposition 1.1 does not hold for nonassociative groupoids. A counterexample is the class of groupoids considered in [14].

Proposition 1.2. A semigroup $\mathbb{S}=\langle S, x y\rangle$ is a TC-semigroup if and only if one of the following conditions holds identically:

(i) $x y=x^{2}$;

(ii) $x y=y^{2}$;

(iii) $x y=y x$ and $x y^{2}=x^{3}$;

(iv) $x y=y x$ and $x y^{2}=x^{2} y$. 
Proof. We prove the "only if" part. At first, note that if $x y$ is not essentially binary, then either $x y=x z$ or $x y=z y$, and consequently the condition (i) or (ii) holds. If $x y$ is essentially binary, then by definition $x y=y x$. Now, consider the polynomial $x y^{2}$. If it is also essentially binary, then $x y^{2}=x^{2} y$, and the condition (iv) holds. If $x y^{2}$ is not essentially binary, then either $x y^{2}=x z^{2}$ or $x y^{2}=z y^{2}$ holds. In the former, $x y^{2}=x^{3}$, so (iii) holds. In the latter $x y^{2}=y^{3}$ and consequently $x^{2} y^{2}=y^{3}$ and, by commutativity, $y^{3}=x^{3}$. Hence, again $x y^{2}=x^{3}$, which proves the "only if" part.

To prove the "if" part, note at first, that if the condition (i) or (ii) is satisfied, then there is no essentially binary polynomial in $\mathbb{S}$, and consequently $S$ is totally commutative ((i) and (ii) are thus trivial cases).

Assume that the condition (iv) holds. Then, it is easy to deduce by simple induction, that $x^{k} y^{m}=x^{k-1} y^{m+1}$ for any $k>m>0$, and in consequence $x^{k} y^{m}=x^{m} y^{k}$. In particular, $\mathrm{S}$ is a TC-semigroup.

It remains to consider the condition (iii). First we note that $x y^{2 k}=$ $x^{3}$ for any $k>0$. Consequently, $x^{m} y^{2 k}$ and $x^{2 k} y^{m}$ are not essentially binary. On the other hand, substituting $x y$ for $x$ in $x y^{2}=x^{3}$, we obtain $x y^{3}=(x y)^{3}=x^{3} y$, and consequently, as above, $x^{k} y^{m}=x^{m} y^{k}$ for any odd $k>m>0$. Hence, every polynomial $x^{k} y^{m}$ either does not depend on one of the variables or is symmetric, thus completing the proof.

\section{Improper semigroups}

Definition. A semigroup $\mathbb{S}=\langle S, x y\rangle$ is proper if $x y$ is essentially binary. Otherwise, it is called improper.

By the proof of Proposition 1.2, $\mathrm{S}$ is improper if and only if the condition (i) or (ii) holds. In particular, every improper semigroup is totally commutative. It is an easy exercise to find normal forms for words in improper semigroups and describe the lattices of subvarieties. Figure 1 shows both the lattices. The subvarieties given by $x y=x, x y=z^{2}, x y=y$ are known respectively as left zero semigroups, constant semigroups, right zero semigroups, and trivial semigroups are at the bottom (see [17]).

Proposition 2.1. Let $\mathbb{S}$ be an improper semigroup. Denote $\varphi(x)=x^{2}$ and $T=\varphi(S) \subseteq S$. Then $\varphi(x)=x$ for every $x \in T$ and either $x y=\varphi(x)$ or $x y=\varphi(y)$. Conversely, given two sets $S \supseteq T$ and a function $\varphi$ of $S$ onto $T$ satisfying $\varphi(x)=x$ for $x \in T$, define $x y=\varphi(x)$ (or $x y=\varphi(y))$. Then $\langle S, x y\rangle$ is an improper semigroup. 


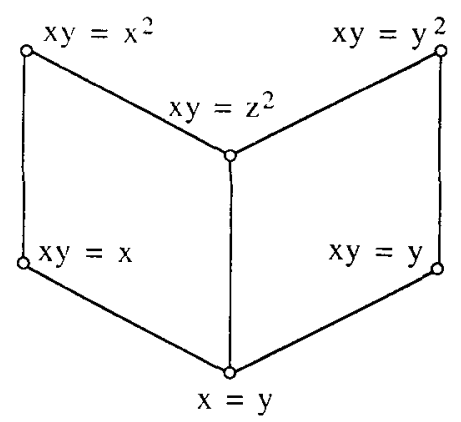

Figure 1

Moreover, $\mathbb{S}$ is a left (or right) zero semigroup if and only if $T=S ; \mathbb{S}$ is a constant semigroup if and only if the cardinality $|T|=1$; and $\mathrm{S}$ is trivial if and only if $|S|=1$.

Proof. This is trivial to prove.

\section{TC-semigroups with $x y^{2}=x^{3}$}

In this section we consider the variety $G$ of semigroups defined by the laws $x y=y x$ and $x y^{2}=x^{3}$ (the case (iii) in Proposition 1.2). All deductions are carried out in $\mathrm{Eq} G$ (the equational theory of $G$ ). In particular, equivalent identities means here equivalent in $\mathrm{Eq} G$. Also $x, y, z$ stand for variables, while $\sigma, \tau, \delta$ stand always for words (terms of semigroups).

At first, we observe that the word $x^{6}$ induces a constant polynomial in every member of $G$. Indeed, from $x y^{2}=x^{3}$ we have $x^{2} y^{2}=x^{6}$ and by commutativity $x^{2}=y^{2} x^{2}=y^{6}$, as required. Hence, in the sequel, we will write $C$ for $x^{6}$.

In turn, we establish some simple laws holding in $G$. We have

(i) $x^{2 k}=C$ for every $k>1$,

(ii) $x^{2 k+1}=x^{3}$ for every $k>1$,

(iii) $C x=x^{3}$,

(iv) $x^{3} y^{3}=x^{3} y=y^{3} x$

Indeed, from $x^{6}=x^{2} y^{2}$ we have $x^{6}=x^{4}$ and (i) follows by simple induction. From (i) we have $x^{2 k+1}=C x$ for $k>1$ which combined with $x^{5}=x\left(x^{2}\right)^{2}=x^{3}$ yields (ii) and (iii). Finally, $x^{3} y^{3}=x^{2} y\left(x y^{2}\right)=x^{5} y$ combined with (ii) yields (iv).

Now, we find normal forms for words in $G$. 
LEMMA 3.1. For every $a_{1}, \ldots, a_{n} \geq 1, \sigma=x_{1}^{a_{1}} \ldots x_{n}^{a_{n}}$ is equal to one and only one of the following words:

(a) $x_{1} \cdots x_{n}$ whenever $a_{1}=\cdots=a_{n}=1$;

(b) $C$ whenever all $a_{1}, \ldots, a_{n}$ are even and either $n>1$ or $a_{1}>2$;

(c) $x_{1}^{2}$ whenever $n=1$ and $a_{1}=2$;

(d) $x_{i_{1}}^{3} x_{i_{2}} \cdots x_{i_{k}}$ when $a_{i_{1}}, \ldots, a_{i_{k}}$ are all odd numbers among $a_{1}, \ldots, a_{n}$ $\left(i_{1}<\cdots<i_{k}\right)$ and $k \geq 1$.

Proof. We prove that $\sigma$ is equal to one of words given in (a) to (d). At first, note that by (i), $C^{2}=C$. Now, using (i) it is easy to see that (b) holds. Also (a) and (c) are trivial. In the remaining case, by (b), $\sigma=$ $C x_{i_{1}}^{b_{1}} \cdots x_{i_{k}}^{b_{k}}$, where $\left\langle b_{1}, \ldots, b_{k}\right\rangle=\left\langle a_{i_{1}}, \ldots, a_{i_{k}}\right\rangle$ consists of all odd numbers among $a_{1}, \ldots, a_{n}$, or $\sigma=x_{1}^{a_{1}} \ldots x_{n}^{a_{n}}$ (if all $a_{i}$ are odd) and then one of $a_{i} \geq 3$ (since otherwise, it would be case (a), not (d)). Using (iii), we see that in any case we have $\sigma=x_{i_{1}}^{c_{1}} \cdots x_{i_{k}}^{c_{k}}$, where all $c_{i}$ are odd and at least one $c_{i} \geq 3$. Applying (iv) we infer that $\sigma=x_{i_{1}}^{3} \cdots x_{i_{k}}$, as asserted.

From rules of deduction applied in this section it is almost obvious that words given in (a) to (d) are pairwise distinct. But the formal proof along this line is rather tedious. An alternate proof is to construct the $G$-free semigroup on $\aleph_{0}$ free generators using forms given in (a) to (d). More precisely, let $S$ be the set of all expressions of the form $x_{i_{1}} \cdots x_{i_{n}}, C, x_{i}^{2}$ and $x_{i_{1}}^{3} x_{i_{2}} \cdots x_{i_{n}}$, where $i_{1}, i_{2}, \ldots, i_{n} \in\{1,2,3, \ldots\}, i_{1}<\cdots<i_{n}$ and $n \geq 1$. We define a binary operation $\circ$ on the set $S$ assuming that the value of $\sigma \circ \tau$ is one of elements of $S$ which corresponds to the formal expression according to the rules given in (a) to (d), commutativity and (iii). Now, it is easy to check that $\mathrm{S}=\langle S, \sigma \circ \tau\rangle$ is a semigroup satisfying $x y=y x$ and $x y^{2}=x^{3}$, that is, belonging to the variety $G$. Since $x_{1}, x_{2}, \cdots \in S$, we see that words given in (a) to (d) induce pairwise distinct polynomials in $\mathbb{S}$, which completes the proof.

Lemma 3.1 shows that each word in $G$ can be reduced to the unique form which we shall call normal. In particular, any identity $\left(\sigma^{\prime}=\tau^{\prime}\right) \notin \mathrm{Eq} G$ is equivalent to some $(\sigma=\tau) \notin \mathrm{Eq} G$, where $\sigma$ and $\tau$ are normal forms for $\sigma^{\prime}$ and $\tau^{\prime}$.

Recall that $\sigma=\tau$ is regular if exactly the same variables appear on both sides.

LEMMA 3.2. If $(\sigma=\tau) \notin \mathrm{Eq} G$ is nonregular and both $\sigma$ and $\tau$ are given in normal forms, then $\sigma=\tau$ is equivalent to the conjunction $\sigma=C$ and $\tau=C$. 
Proof. If $\sigma=\tau$ is of the form $x^{k}=y^{n}$, then the result is immediate. Otherwise, by Lemma 3.1 we can assume that $\sigma=\tau$ is of the form $\sigma=x \delta$, where $x$ is a variable appearing neither in $\sigma$ nor in $\delta$ (the empty word $\delta$ is not excluded). Substituting $x=\delta^{3}$ we get $\sigma=\delta^{4}$, and by (i), $\sigma=C$, proving the lemma.

In the sequel we use the following additional notation (for every $n \geq 1$ ):

$$
\begin{aligned}
& i_{n}=x_{1} x_{2} \cdots x_{n} \\
& t_{n}=x_{1}^{3} x_{2} \cdots x_{n} ; \\
& \Sigma_{0}=\left\{x y=y x, x(y z)=(x y) z, x y^{2}=x^{3}\right\} ; \\
& {\left[I_{1}, \ldots, I_{n}\right]=\operatorname{Mod}\left(\Sigma_{0} \cup\left\{I_{1}, \ldots, I_{n}\right\}\right) .}
\end{aligned}
$$

Of course, $\Sigma_{0}$ is an equational base for the variety $G$, and $\left[I_{1}, \ldots, I_{n}\right]$ is the subvariety of $G$ defined by the additional laws $I_{1}, \ldots, I_{n}$.

We will use also the following equivalences, which are easy consequences of (i) to (iv) ( $\leftrightarrow$ stands here for "is equivalent to $\cdots$ in Eq $G$ "):

(v) $x^{2}=x \leftrightarrow x=C$;

(vi) $x^{3}=x^{2} \leftrightarrow\left(x^{2}=C\right.$ and $\left.x^{3}=C\right)$;

and for every $n \geq 1$,

(vii) $t_{n}=C \leftrightarrow x^{3}=C$;

(viii) $i_{n}=C \leftrightarrow\left(x^{3}=C\right.$ and $\left.i_{n}=t_{n}\right)$.

Indeed, $x^{2}=x$ implies $x^{3}=x^{2}$ and $x^{4}=x^{3}$, which by (i) yields $x=C$. The converse implication also holds, for $C^{2}=C$. Similarly we prove (vi). Substituting $x_{2}=\cdots=x_{n}=C$ in $t_{n}=C$ we get by (iii) and (ii), $x_{1}^{3}=C$. Conversely, substituting $x=x_{1} \cdots x_{n}$ in $x^{3}=C$ we get, by (iv), $t_{n}=C$, proving (vii). Finally, $i_{n}=C$ implies $x^{3}=C$ (if $n<3$ we apply an appropriate substitution; otherwise $i_{n}=C$ implies $t_{n-2}=C$ and we use (vii)). In turn, $i_{n}=C$ and $x^{3}=C$ together imply, by (vii), $t_{n}=C$ and consequently, $i_{n}=t_{n}$. Conversely, $x^{3}=C$ in view of (vii) yields $t_{n}=C$, which combined with $i_{n}=t_{n}$ yields $i_{n}=C$, thus proving (viii).

Now we prove

Lemma 3.3. If $X$ is a subvariety of $G$, then $X=\operatorname{Mod}\left(\Sigma_{0} \cup \Sigma\right)$, where $\Sigma \subseteq\left\{i_{n}=t_{n}, x^{2}=C, x^{3}=C\right\}$ for some $n \geq 1$.

Proof. Let $\Sigma^{*}=\mathrm{Eq} X$. By assumption $\Sigma^{*} \supseteq \Sigma_{0}$. Our aim is to show that $\Sigma^{*}$ can be reduced to $\Sigma_{0} \cup \Sigma$. The first step is that in view of Lemmas 3.1 and 3.2 all identities in $\Sigma^{*}$ other than those in $\Sigma_{0}$ can be assumed to be of the following forms $(n \geq 1): i_{n}=t_{n} ; x^{2}=x ; x^{3}=x^{2}$ (these are all possible regular identities assuming normal forms); $i_{n}=C ; t_{n}=C$; and $x^{2}=C$. 
Further, $x^{2}=x$ and $x^{3}=x^{2}$ can be omitted in view of (v) and (vi). In turn, all $t_{n}=C$ can be replaced by $x^{3}=C$ in view of (vii). Finally, using (viii) we replace by $x^{3}=C$ and $i_{n}=t_{n}$ all identities $i_{n}=C$. This shows that $\Sigma^{*}$ can be reduced to $\Sigma_{0} \cup \Sigma$, where $\Sigma \subseteq\left\{i_{n}=t_{n}(n \geq 1), x^{2}=C\right.$, $\left.x^{3}=C\right\}$ with $n$ running over positive integers. To complete the proof it is enough to observe that $i_{k}=t_{k}$ implies obviously $i_{n}=t_{n}$ for all $n \geq k$, and therefore any set of identities $i_{n}=t_{n}$ can be replaced by one with the least index.

As a consequence of Lemma 3.3 we have the following description of all subvarieties of $G$.

Proposition 3.4. Each subvariety of $G$ is finitely based and is equal to one and only one of the following varieties:

$G_{k}=\left[i_{k}=t_{k}\right]$ for some $3 \leq k \leq \omega$;

$G_{k}^{*}=\left[i_{k}=t_{k}, x^{2}=C\right]$ for some $1 \leq k \leq \omega$;

$G_{k}^{0}=\left[i_{k}=t_{k}, x^{3}=C\right]$ for some $3 \leq k \leq \omega$;

$G_{k}^{* 0}=\left[i_{k}=t_{k}, x^{2}=C, x^{3}=C\right]$ for some $1 \leq k \leq \omega$, where $i_{\omega}=t_{\omega}=x$.

REMARK. The convention $i_{\omega}=t_{\omega}=x$ is introduced here for the number of cases distinguished to be as small as possible. The identity $i_{\omega}=t_{\omega}$ reads simply $x=x$, and as a matter of fact can be omitted. In particular, $G_{\omega}=G$. We note also that $G_{1}^{* 0}=\left[x=x^{3}, x^{2}=C, x^{3}=C\right]=[x=C]=[x=y]$ is the variety of trivial (one-element) semigroups, $G_{2}^{* 0}=\left[x y=x<3 y, x^{2}=\right.$ $\left.C, x^{3}=C\right]=[x y=C]$ is the variety of constant semigroups, while $G_{1}^{*}=$ $\left[x=x^{3}, x^{2}=C\right]$ is equivalent to the variety of Boolean groups.

Proof. Since $i_{\omega}=t_{\omega}=x$, we have taken into account all possible subsets of $\left\{i_{k}=t_{k}, x^{2}=C, x^{3}=C\right\}$ for all $k \geq 3$. For $k=2, i_{2}=t_{2}$ is $x y=x^{3} y$ and implies $x^{2}=C$. Also, for $k=1, i_{1}=t_{1}$ is $x=x^{3}$ and implies $x^{2}=C$. This means that for $k=1,2$ the bases $\left[i_{k}=t_{k}\right]$ and $\left[i_{k}=t_{k}, x^{3}=C\right]$ need not be taken into account. Hence, the first part of the proposition is a simple consequence of Lemma 3.3. In turn, the fact that all listed varieties are pairwise distinct is an immediate consequence of the following proposition.

Proposition 3.5. If $X$ is a subvariety of $G$, then $\mathrm{Eq} X$ is a decidable theory and solutions for corresponding word problems are given by the following list, where each of the equational bases given consists of all nontrivial identities of $X$ using normal forms: 


$$
\begin{aligned}
& G_{k}=\left[i_{n}=t_{n}(n \geq k)\right] \\
& G_{k}^{*}=\left[i_{n}=t_{n},(n \geq k), x^{2}=C\right] \\
& G_{k}^{0}=\left[i_{n}=t_{n}(n \geq k), i_{n}=C(n \geq k), t_{n}=C(n \geq 1)\right] \\
& G_{k}^{* 0}=\left[i_{n}=t_{n}(n \geq k), i_{n}=C(n \geq k), t_{n}=C(n \geq 1), x^{2}=C\right] .
\end{aligned}
$$

Proof. It is of course enough in every case to construct the semigroup of words, analogously, as in the proof of Lemma 3.1, using the identities given in square brackets and then check that this is the $X$-free semigroup on the set $\left\{x_{1}, x_{2}, x_{3}, \ldots\right\}$. This is rather long, but completely routine and therefore is left to the reader.

Notice that the first part of the proposition is an immediate consequence of the general result of Evans [6], combined, of course, with Proposition 3.4.

From Propositions 3.4 and 3.5 it is easy to describe the lattice of subvarieties of $G$.

COROllary 3.6. For every subvariety $X$ of $G$, and for every $k$ (as indicated in Proposition 3.4):

(a) $G_{k} \supseteq X$ if and only if $X=G_{m}$ or $X=G_{m}^{*}$ or $X=G_{m}^{0}$ or $X=G_{m}^{* 0}$ for some $m \leq k$;

(b) $G_{k}^{*} \supseteq X$ if and only if $X=G_{m}^{*}$ or $X=G_{m}^{* 0}$ for some $m \leq k$;

(c) $G_{k}^{0} \supseteq X$ if and only if $X=G_{m}^{0}$ or $X=G_{m}^{* 0}$ for some $m \leq k$;

(d) $G_{k}^{* 0} \supseteq X$ if and only if $X=G_{m}^{* 0}$ for some $m \leq k$.

Proof. We prove (b). By Proposition 3.4, $G_{k}^{*}=\left[i_{k}=t_{k}, x^{2}=C\right]$. If $X=G_{m}^{*}$ or $X=G_{m}^{* 0}$ for some $m \leq k$, then in view of Proposition 3.5 both $i_{k}=t_{k}$ and $x^{2}=C$ hold in $X$, which means obviously that $G_{k}^{*} \supseteq X$. Otherwise, as it is easy to check, either $i_{m}=t_{m}$ or $x^{2}=C$ does not hold in $X$, and consequently, neither does $G_{k}^{*} \supseteq X$, proving (b). The proof in the remaining cases is analogous.

By Corollary 3.6 the four families of subvarieties of $G$ given in Proposition 3.4 form infinite chains in the lattice of subvarieties of $G$ (ordered by the natural order of indices) which are connected by a diamond pattern: $G_{n} \supseteq G_{n}^{*}, G_{n}^{0} \supseteq G_{n}^{* 0}$ (see Figure 2).

Another important corollary is that concerning free spectra of subvarieties of $G$ (sizes of free algebras). As often occurs, it is however more convenient to describe $p_{n}$-sequences of these subvarieties rather than free spectra. Recall that if we denote by $p_{n}=p_{n}(X)$ the number of essentially $n$-ary polynomials over the $X$-free semigroup on $\aleph_{0}$ free generators, then the cardinality 


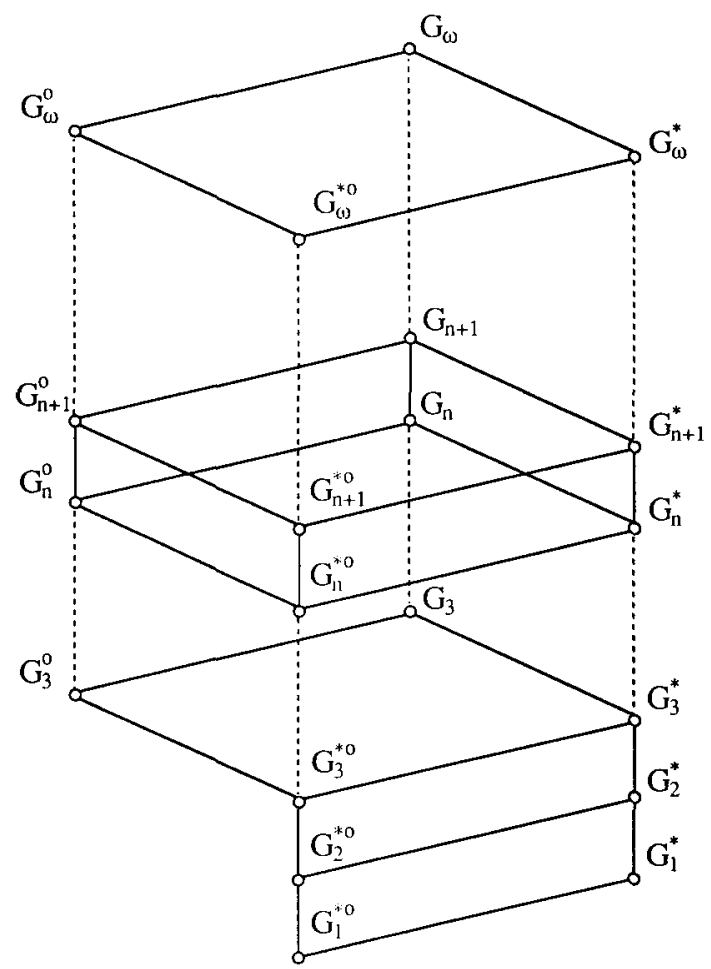

Figure 2

$s_{n}=s_{n}(X)$ of the $X$-free semigroup on $n$ free generators is given by

$$
s_{n}=\sum_{k=0}^{n}\left(\begin{array}{l}
n \\
k
\end{array}\right) p_{k}
$$

$\left(p_{0}(X)\right.$ is here the number of constants, that is, constant unary polynomials; see $[5,13,16]$ and [9] for more details on this topic).

COROllary 3.7. For every subvariety $X$ of $G, p_{0}(X)=1$ and $p_{n}(X)$ for $n \geq 1$ is given in Table 1. Moreover, $p_{n}\left(G_{2}^{*}\right)=p_{n}\left(G_{\omega}^{0}\right), p_{n}\left(G_{1}^{*}\right)=p_{n}\left(G_{\omega}^{* 0}\right)$ for all $n \geq 0$, and $p_{n}\left(G_{2}^{* 0}\right)=p_{n}\left(G_{1}^{* 0}\right)=0$ for $n \geq 1$, except for $p_{1}\left(G_{2}^{* 0}\right)=1$. In particular,

$$
\begin{aligned}
& s_{n}\left(G_{\omega}\right)=2^{n+1}+n-1, \\
& s_{n}\left(G_{\omega}^{*}\right)=2^{n+1}-1, \\
& s_{n}\left(G_{2}^{*}\right)=s_{n}\left(G_{\omega}^{0}\right)=2^{n}+n, \\
& s_{n}\left(G_{1}^{*}\right)=s_{n}\left(G_{\omega}^{* 0}\right)=2^{n}
\end{aligned}
$$

for all $n \geq 0$. 
TABLE 1.

\begin{tabular}{|c|c|c|c|}
\hline $\begin{array}{c}X \\
\text { (for } 3 \leq k \leq \omega)\end{array}$ & $p_{1}(X)$ & $\begin{array}{c}p_{n}(X) \\
\text { (for } 2 \leq n \leq k)\end{array}$ & $\begin{array}{c}p_{n}(X) \\
\text { (for } k \leq n)\end{array}$ \\
\hline$G_{k}$ & 3 & 2 & 1 \\
$G_{k}^{*}$ & 2 & 2 & 1 \\
$G_{k}^{0}$ & 2 & 1 & 0 \\
$G_{m}^{* 0}$ & 1 & 1 & 0 \\
\hline
\end{tabular}

Proof. This is immediate from Proposition 3.5.

Finally, it is also interesting to know which of these varieties is generated (and in consequence, which of these $p_{n}$-sequences is represented) by a finite semigroup. We have

COROLLARY 3.8. If $X$ is a subvariety of $G$, then $X$ is generated by a finite semigroup if and only if $X \neq G_{\omega}, G_{\omega}^{*}, G_{\omega}^{0}$ or $G_{\omega}^{* 0}$.

Proof. Assume that $X$ is generated by a $k$-element semigroup $\mathrm{S}$. Then, we show that the identity $i_{2 k+2}=t_{2 k+2}$ holds in $\mathrm{S}$. Indeed, consider the value $v$ of $t_{2 k+2}=x_{1}^{3} x_{2} \cdots x_{2 k+2}$ for some elements $s_{1}, s_{2}, \ldots, s_{2 k+2}$ of $S$. Clearly, since the cardinality $|\mathbb{S}|=k$, we have $s_{i}=s_{j}=s_{m}$ for some $i, j, m \in\{2,3, \ldots, 2 k+2\}$. Hence, $v=s_{1}^{3} s^{3} r$, where $s=s_{i}$ and $r$ is the product of the remaining elements $s_{n}$. By Lemma 3.1, $v=s_{1}^{3} s r=s^{3} s_{1} r$. In turn, the value $w$ of $i_{2 k+2}=x_{1} x_{2} \cdots x_{2 k+2}$ for $s_{1}, s_{2}, \ldots, s_{2 k+2}$ is $w=$ $s_{1} s^{3} r=s^{3} s_{1} r=v$, proving that $i_{2 k+2}=t_{2 k+2}$.

This shows, in view of Proposition 3.5, that the variety $X$ generated by $\mathrm{S}$ is different from $G_{\omega}, G_{\omega}^{*}, G_{\omega}^{0}$ and $G_{\omega}^{* 0}$, since in these varieties no identity $i_{n}=t_{n}$ holds.

Conversely, if for example, $X=G_{k}$ for some $k<\omega$, then let $\mathbb{S}_{k}$ be the $G_{k}$ free semigroup on $k$ free generators. By Corollary 3.7, $\mathrm{S}_{k}$ is finite. The identities in $n \leq k$ variables are the same in $\mathbb{S}_{k}$ and in $G_{k}$. On the other hand $i_{n}=x_{1} x_{2} \cdots x_{n}$ for $n>k$ yields $C$ if we substitute $x_{1}=\cdots=x_{n}=$ $C$, and $x^{3}$, if we substitute $x_{1}=x$ and $x_{2}=\cdots=x_{n}=C$ (for $C x=x^{3}$ ). This means that $i_{n}$ is essentially $n$-ary in $\mathbb{S}_{k}$, and in consequence that identities in more than $k$ variables are also the same in $\mathbb{S}_{k}$ and in $G_{k}$. It 
follows that the semigroup $\mathbb{S}_{k}$ generates the variety $G_{k}$. Similarly we prove that any $X \neq G_{\omega}, G_{\omega}^{*}, G_{\omega}^{0}$ or $G_{\omega}^{* 0}$ is generated by a finite semigroup, which completes the proof.

\section{TC-semigroups with $x y^{2}=x^{2} y$}

In this section we consider the variety $H$ of commutative semigroups defined by the law $x y^{2}=x^{2} y$. Results and proofs are to a large extent similar to those in Section 3. So some analogous details will be now omitted. Also we use the same notation as in Section 3 except that now, of course

$$
\Sigma_{0}=\left\{x y=y x, x(y z)=(x y) z, x y^{2}=x^{2} y\right\}
$$

and equivalent means equivalent in $\mathrm{Eq} H$.

As previously, we start by finding normal forms for words in $H$.

LemMA 4.1. The unary words $x, x^{2}, x^{3}, x^{4}$ are pairwise distinct in $H$, and $x^{n}=x^{4}$ for any $n \geq 4$.

If $n \geq 2$, then for every $a_{1}, \ldots, a_{n} \geq 1, \sigma=x_{1}^{a_{1}} \ldots x_{n}^{a_{n}}$ is equal to one and only one of the following words:

(a) $i_{n}=x_{1} x_{2} \cdots x_{n}$ whenever $a_{1}=\cdots=a_{n}=1$;

(b) $b_{n}=x_{1}^{2} x_{2} \cdots x_{n}$ whenever $\Sigma a_{i}=n+1$;

(c) $t_{n}=x_{1}^{3} x_{2} \cdots x_{n}$, otherwise.

Proof. At first, we have $x^{2} y^{2}=x^{4} y$, and on the other hand $x^{2} y^{2}=$ $x\left(x y^{2}\right)=x^{3} y$. Hence, $x^{3} y=x^{4} y$, and by simple induction, $x^{3} y=x^{n} y$ for every $n \geq 4$. In particular, $x^{4}=x^{n}$ for every $n \geq 4$. Using $x^{2} y^{2}=x^{4} y$ a number of times, we find that $x^{r} y^{s}=x^{4} y=x^{3} y$, provided $r+s \geq 4(r, s \geq$ 1). It follows easily that $x_{1}^{a_{1}} \cdots x_{1}^{a_{n}}=x_{1}^{3} x_{2} \cdots x_{n}$, whenever $\sum a_{i} \geq n+2$.

Condition (b) is immediate from the defining identity $x y^{2}=x^{2} y$, and (a) is trivial.

Now, analogously to the proof of Lemma 3.1, using words given in Lemma 4.1, we construct the $H$-free semigroup on the set $\left\{x_{1}, x_{2}, \ldots\right\}$, which proves that the listed words are pairwise distinct and can be used as normal forms, as asserted.

Note that in the present case the identities defining $H$ are all regular.

Lemma 4.2. Let $\mathbb{S} \in H$. Then $\mathbb{S}$ satisfies a nonregular identity $\sigma=\tau$ if and only if $\mathrm{S}$ has a constant $C$. If so, the constant is unique, determined by $C=x^{4}$, and $C x=C$ holds. Moreover, $\sigma=\tau$ is equivalent to the conjunction $\sigma=C$ and $\tau=C$. 
Proof. If $\mathrm{S}$ has a constant $C$, then by definition some nonregular identity $\sigma=C$ holds. Substituting $x^{4}$ for every variable occurring in $\sigma$ we obtain, by Lemma $4.1, x^{4}=C$. This implies $x^{5}=C x$, and since $x^{5}=x^{4}$, we have $C=C x$, which shows also that the constant is unique.

Conversely, if a nonregular identity holds in $\mathrm{S}$, then by Lemma 4.1 we can assume that either some identity $\delta z=\tau$ holds, where $z$ is a variable not occurring in $\tau$ and $\delta$, or $x^{n}=y^{m}$ holds. From the latter, in any case, it is easy to infer $x^{4}=y^{4}$, as required. From the former, substituting $x$ for all variables in $\delta$ and $\tau$ (and multiplying both the sides by $x^{4}$, if necessary) we obtain, in view of Lemma 4.1, that $x^{3} z=x^{4}$. Since $x^{3} z=z^{3} x$, it follows that $x^{4}=z^{4}$, and consequently, $x^{4}=C$.

Finally, substituting $z=C$ in $\delta z=\tau$, we get $C=\tau$ (for we have proved already that $C x=C$ ), and in consequence, $\sigma=\delta z=C$ too. Since in the latter case obviously $x^{n}=y^{m}=C$, the proof is completed.

As an analogue of Lemma 3.3 we have

LEMMA 4.3. If $X$ is a subvariety of $H$, then $X=\operatorname{Mod}\left(\Sigma_{0} \cup \Sigma\right)$, where $\Sigma \subseteq\left\{i_{n}=b_{n}, b_{m}=t_{m}, x^{3}=x^{4}, x^{4}=y^{4}\right\}$ for some $n, m \geq 1$.

Proof. First we establish some equivalences we will use subsequently:

(i) $x^{4}=x \leftrightarrow x^{2}=x$;

(ii) $x^{4}=x^{2} \leftrightarrow x^{3}=x^{2}$ for every $n \geq 1$;

(iii) $i_{n}=t_{n} \leftrightarrow i_{n}=b_{n}$.

Under the additional assumption, that there is a constant $C=x^{4}$ (according to Lemma 4.2), we have $t_{n}=C$ for every $n \geq 2$, and for every $n \geq 1$

(iv) $i_{n}=C \leftrightarrow i_{n}=b_{n}$.

In order to prove (i) note that $x^{4}=x$ yields $x^{5}=x^{2}$, that is, in view of Lemma 4.1, $x^{4}=x^{2}$, and further $x^{4}=x^{3}$, and in consequence, $x^{2}=x$. Similarly, we prove the converse implication, and analogously (ii) and (iii).

Assume that $x^{4}=C$. Then, by Lemmas 4.1 and $4.2, t_{n}=x_{1}^{3} x_{2} \cdots x_{n}=$ $x_{1}^{4} x_{2} \cdots x_{n}=C x_{2} \cdots x_{n}=C$, provided $n \geq 2$. If now $i_{n}=C$, then also $b_{n}=C$, that is $i_{n}=b_{n}$. Conversely, $i_{n}=b_{n}$ implies $b_{n}=t_{n}=C$ and consequently, $i_{n}=C$, proving (iv).

Now $\Sigma^{*}=\mathrm{Eq} X$ is, of course, an equational base for $X$. We wish to show that $\Sigma^{*}$ can be replaced by $\Sigma_{0} \cup \Sigma$ with

$$
\Sigma \subseteq\left\{i_{n}=b_{n}, b_{m}=t_{m}, x^{3}=x^{4}, x^{4}=y^{4}\right\},
$$

where $n$ and $m$ run over positive integers. In view of Lemmas 4.1 and 4.2 
we can assume that $\Sigma^{*}$ contains merely identities of the forms

$$
x^{4}=x, \quad x^{4}=x^{2}, \quad x^{4}=x^{3}, \quad i_{n}=b_{n}, \quad i_{n}=t_{n}, \quad b_{n}=t_{n}
$$

(which are regular identities), and if $x^{4}=y^{4} \in \mathrm{Eq} X$,

$$
x^{4}=y^{4}, x^{3}=C, i_{n}=C, b_{n}=C,
$$

and in addition those of $\Sigma_{0}$.

By (i) and (ii), $x^{4}=x$ and $x^{4}=x^{2}$ can be replaced by $b_{1}=i_{1}$ and $t_{1}=b_{1}$, respectively. In turn, by (iii), $i_{n}=t_{n}$ can be replaced by $i_{n}=b_{n}$. It follows that, if $x^{4}=y^{4} \notin \mathrm{Eq} X$, that is, by Lemma 4.2, if all identities in $\mathrm{Eq} X$ are regular, then our assertion is true. Hence, assume that $x^{4}=$ $y^{4} \in \mathrm{Eq} X$. Then, as we have shown above, $t_{n}=x^{4}=C$ for every $n \geq 2$. Hence, $x^{3}=C$ can be replaced by $x^{3}=x^{4}$, and $b_{n}=C$, by $b_{n}=t_{n}$, for $n \geq 2$. But $b_{1}=x_{1}^{2}=C$ is equivalent to $x^{2}=x^{3}$ (see (iii)) and thus can be replaced by $b_{n}=t_{n}$ (for $n=1$ ), too. Finally, by (iv), $i_{n}=C$ can be replaced by $i_{n}=b_{n}$, which completes the first part of the proof.

Now, as in the proof of Lemma 3.3, we observe that $i_{k}=b_{k}$ implies $i_{n}=b_{n}$ for all $n \geq k$, and $b_{m}=t_{m}$ implies $b_{n}=t_{n}$ for all $n \geq m$, thus proving Lemma 4.3 .

Now we state our propositions and corollaries analogous to those in Section 3. We omit proofs for they are essentially the same as corresponding proofs in Section 3.

Proposition 4.4. Each subvariety of $H$ is finitely based and is equal to one and only one of the following varieties:

$H_{k, m}=\left[i_{k}=b_{k}, b_{m}=t_{m}\right]$ for some $\omega \geq k>m \geq 3$ or $k=m=\omega$;

$H_{k, m}^{*}=\left[i_{k}=b_{k}, b_{m}=t_{m}, x^{3}=x^{4}\right]$ for some $\omega \geq k>m \geq 1$ or $k=m=\omega$ or $k=m=1$;

$H_{k, m}^{0}=\left[i_{k}=b_{k}, b_{m}=t_{m}, x^{4}=y^{4}\right]$ for some $\omega \geq k>m \geq 3$ or $k=m=\omega$;

$H_{k, m}^{* 0}=\left[i_{k}=b_{k}, b_{m}=t_{m}, x^{3}=x^{4}=y^{4}\right]$ for some $\omega \geq k>m \geq 1$ or $k=m=\omega$ or $k=m=1$,

where $i_{\omega}=b_{\omega}=x$.

REMARK. In the sequel, $k$ and $m$ are assumed to run over the sets indicated in Proposition 4.4.

Proposition 4.5. If $X$ is a subvariety of $H$, then $\mathrm{Eq} X$ is a decidable theory and solutions for corresponding word problems are given by the following list of subvarieties of $H$, where each of equational bases given consists of all 
nontrivial identities of $X$ using normal forms:

$$
\begin{aligned}
& H_{k, m}=\left[i_{n}=b_{n}(n \geq k), b_{n}=t_{n}(n \geq m)\right] ; \\
& H_{k, m}^{*}=\left[i_{n}=b_{n}(n \geq k), b_{n}=t_{n}(n \geq m), x^{3}=x^{4}\right] ; \\
& H_{k, m}^{0}=\left[i_{n}=C(n \geq k), b_{n}=C(n \geq m), t_{n}=C(n \geq 2), x^{4}=C\right] ; \\
& H_{k, m}^{* 0}=\left[i_{n}=C(n \geq k), b_{n}=C(n \geq m), t_{n}=C(n \geq 1), x^{4}=C\right] .
\end{aligned}
$$

(For $H_{k, m}^{0}$ and $H_{k, m}^{* 0}$ we have in addition identities $i_{n}=b_{n}, i_{n}=t_{n}$, $b_{n}=t_{n}$ which trivially follow from those given and therefore are omitted.)

COROLlary 4.6. For every subvariety $X$ of $H$ and for every $k$ and $m$ (as indicated in Proposition 4.4), we have

(a) $H_{k, m} \supseteq X$ if and only if $X=H_{r, s}$ or $X=H_{r, s}^{*}$ or $X=H_{r, s}^{0}$ or $X=H_{r, s}^{* 0}$ for some $r \leq k$ and $s \leq m$,

(b) $H_{k, m}^{*} \supseteq X$ if and only if $X=H_{r, s}^{*}$ or $X=H_{r, s}^{* 0}$ for some $r \leq k$ and $s \leq m$,

(c) $H_{k, m}^{0} \supseteq X$ if and only if $X=H_{r, s}^{0}$ or $X=H_{r, s}^{* 0}$ for some $r \leq k$ and $s \leq m$,

(d) $H_{k, m}^{* 0} \supseteq X$ if and only if $H_{r, s}^{* 0}$ for some $r \leq k$ and $s \leq m$.

By Corollary 4.6, the four families of subvarieties given in Proposition 4.4 form infinite sublattices of the lattice of subvarieties of $H$, which are connected, similarly as in the lattice of subvarieties of $G$ Section 3 , by a diamond pattern: $H_{k, m} \supseteq H_{k, m}^{*}, H_{k, m}^{0} \supseteq H_{k, m}^{* 0}$. These four sublattices are isomorphic to the lattices of pairs of indices $(k, m)$ ordered by $(k, m) \geq$ $(r, s)$ if and only if $k \geq r$ and $m \geq s$. One of them is the lattice of subvarities of $H_{k, m}^{* 0}$ given in Figure 3, and is isomorphic to the lattice formed by the family $H_{k, m}^{*}$ (this, however, is not the lattice of subvarieties of $H_{\omega, \omega}^{*}$, since $H_{k, m}^{*} \supseteq H_{r, s}^{* 0}$ for all $\left.r, s\right)$. Two other sublattices are isomorphic to that in Figure 3 without the two bottom elements. Hence, the lattice of subvarieties of $H$ can be presented schematically as in Figure 4.

Note that, $H_{1,1}^{* 0}=\left[x=x^{2}=x^{3}=x^{4}=y^{4}\right]=[x=y]=G_{1}^{* 0}$ is the variety of trivial semigroups, $H_{1,1}^{*}=\left[x=x^{2}=x^{3}=x^{4}\right]=\left[x=x^{2}\right]$ is the variety 


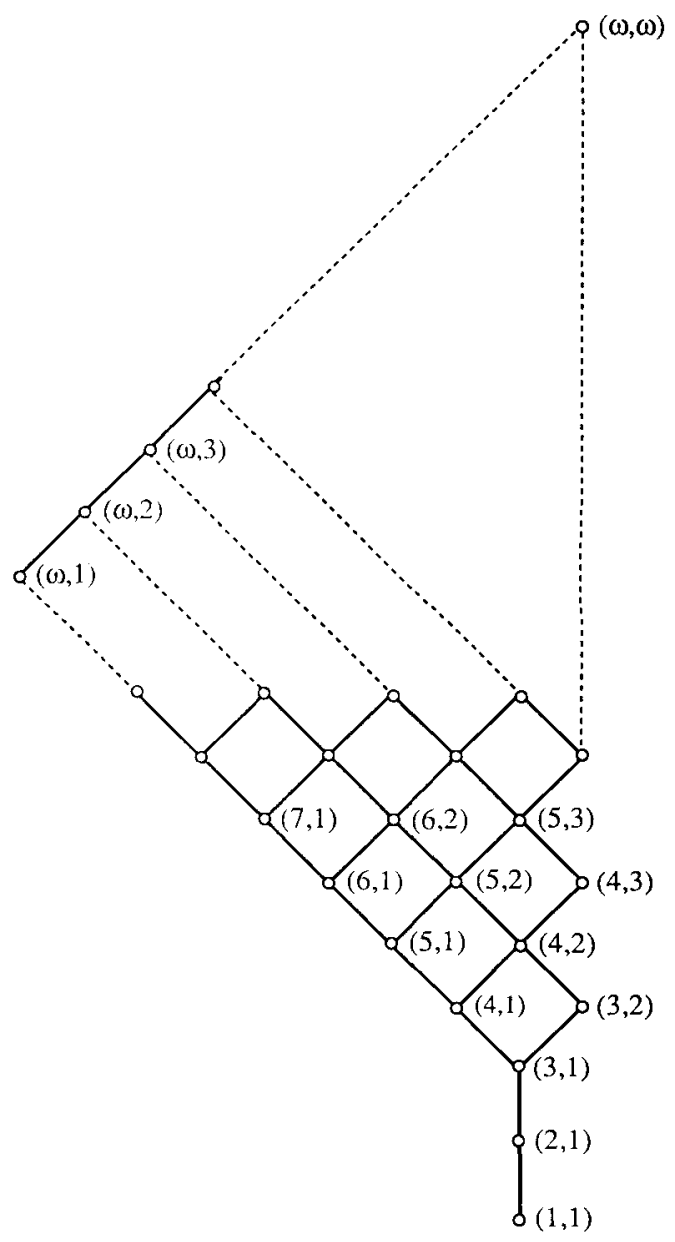

Figure 3

of semilattices, and $H_{2,1}^{* 0}=\left[x y=x^{2} y, x^{2}=x^{3}=x^{4}\right]=[x y=C]=G_{2}^{* 0}$ is the variety of constant semigroups.

Corollary 4.7. For every subvariety $X$ of $H$ the number $p_{n}(X)$ is given in Table 2.

Moreover, for every $1 \leq k \leq \omega, p_{0}\left(H_{k, 1}^{*}\right)=0, p_{n}\left(H_{k, 1}^{*}\right)=2$ for $0<n<$ $k$, and $p_{n}\left(H_{k, 1}^{*}\right)=1$, otherwise. Also, $p_{n}\left(H_{k, 1}^{* 0}\right)=1$ for $0 \leq n<k$, and 


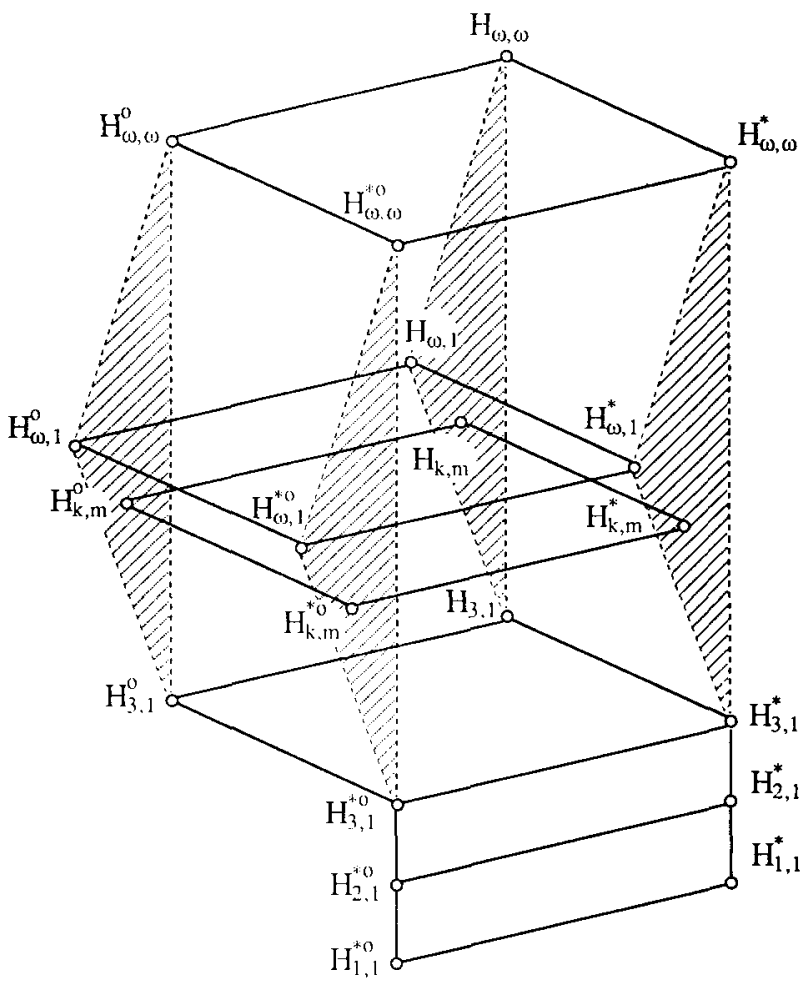

Figure 4

TABLE 2.

\begin{tabular}{|c|c|c|c|c|c|}
\hline $\begin{array}{c}X \\
(\text { for } m \geq 2)\end{array}$ & $p_{0}(X)$ & $p_{1}(X)$ & $\begin{array}{c}p_{n}(X) \\
(1<n<m)\end{array}$ & $\begin{array}{c}p_{n}(X) \\
(m \leq n<k)\end{array}$ & $\begin{array}{c}p_{n}(X) \\
(k \leq n<\omega)\end{array}$ \\
\hline$H_{k, m}$ & 0 & 4 & 3 & 2 & 1 \\
$H_{k, m}^{*}$ & 0 & 3 & 3 & 2 & 1 \\
$H_{k, m}^{0}$ & 1 & 3 & 2 & 1 & 0 \\
$H_{k, m}^{* 0}$ & 1 & 2 & 2 & 1 & 0 \\
\hline
\end{tabular}

$$
\begin{gathered}
p_{n}\left(H_{k, 1}^{* 0}\right)=0, \text { otherwise. In particular, } \\
s_{n}\left(H_{\omega, \omega}\right)=3 \cdot 2^{n}+n-3 \\
s_{n}\left(H_{\omega, \omega}^{*}\right)=3 \cdot 2^{n}-3
\end{gathered}
$$




$$
\begin{aligned}
& s_{n}\left(H_{\omega, \omega}^{0}\right)=2^{n+1}+n-1, \\
& s_{n}\left(H_{\omega, \omega}^{* 0}\right)=2^{n+1}-1, \text { for all } n \geq 0 .
\end{aligned}
$$

COROLlary 4.8. If $X$ is a subvariety of $H$, then $X$ is generated by a finite semigroup if and only if $X \neq H_{\omega, m}, H_{\omega, m}^{*}, H_{\omega, m}^{0}$ and $H_{\omega, m}^{* 0}$ for all $m$.

Finally, combining Corollaries 3.6, 3.8, 4.6 and 4.8 (see Figures 2 and 4) we have

COROLlary 4.9. Let $X$ be any variety of totally commutative semigroups. Then the lattice of subvarieties of $X$ is finite if and only if the variety $X$ is generated by a finite semigroup.

\section{Hyperidentities}

A hyperidentity is formally the same as an ordinary identity but we say that an algebra $\mathbb{A}$ satisfies the hyperidentity $\sigma=\tau$ if and only if $\sigma=\tau$ holds for every choice of polynomials of $\mathbb{A}$ to be substituted for operation symbols appearing in $\sigma$ and $\tau$ (only the arity, of course, has to coincide (see [18]). For example, any idempotent algebra $\mathbb{A}$ satisfies the hyperidentity $F(x, x)=x$, and more generally, $G(x, x, \ldots, x)=x$. But if we consider the hyperidentity

$$
F(x, y)=F(y, x)
$$

(hypercommutativity), then according to this definition, no nontrivial algebra satisfies it, since the binary projection $e(x, y)=x$ (which is by definition a polynomial for every nontrivial algebra) substituted for $F$ yields $x=y$.

On the other hand, in [1 and 3] a weaker definition was considered, not requiring $\sigma=\tau$ to hold for all polynomials. It is reasonable therefore to consider hyperidentity with regard to certain predescribed sets of polynomials; in particular, as suggested at the beginning of this paper, to take into account only essential polynomials (depending on all the variables).

Consider from this point of view, the identities of commutative semigroups, (2) and

$$
F(F(x, y), z)=F(x, F(y, z)),
$$

and let us call groupoids satisfying these two identities for all their essentially binary polynomials hypersemigroups. Then we have the following characterization. 
Proposition 5.1. A proper groupoid A is a hypersemigroup if and only if $\mathbb{A}$ is a totally commutative semigroup. In particular, every essentially binary polynomial in a TC-semigroup generates a TC-semigroup.

Proof. At first, assume that $\mathbb{A}$ is a $T C$-semigroup. Then observe that according to Lemmas 3.1 and 4.1 , every essentially binary polynomials in $\mathrm{A}$ is of the form $f(x, y)=x^{k} y$ for some $k \leq 3$, and satisfies $f(x, y)=$ $x^{k} y=y^{k} x=f(y, x)$. This yields also

$$
f(f(x, y), z)=\left(x^{k} y\right) z^{k}=x^{k} y z^{k}
$$

and

$$
f(x, f(y, z))=x^{k}\left(y z^{k}\right)=x^{k} y z^{k}
$$

which shows that $A$ is a hypersemigroup.

Conversely, if a groupoid $\mathbb{A}=\langle A, x y\rangle$ is a hypersemigroup, then since $x y$ is essentially binary (A is proper), $F(x, y)=x y$ satisfies (2) and (3), and consequently $\mathbb{A}$ is a semigroup. In addition, since every essentially binary polynomial of $\mathbb{A}$ satisfies (2), $\mathbb{A}$ is totally commutative, completing the proof.

The assumption in Proposition 5.1 that $\mathrm{A}$ is proper, is essential, since there are improper groupoids which are not associative. (These are all groupoids defined by $x y=f(x)$ for any $f(x)$ with $f(f(x)) \neq f(x))$

In connection with the results of this paper, it would be interesting, but as it seems, more difficult, to characterize all groupoids which for every essentially binary polynomial satisfy (1) (or respectively (2)).

\section{References}

[1] I. Aczél, 'Proof of a theorem on distributive type hyperidentities', Algebra Universalis 1 (1971), 1-6.

[2] A. P. Birjukov, 'Varieties of idempotent semigroups', Algebra i Logika 9 (1970), 255273.

[3] V. D. Belousov, 'Systems of quasigroups with generalized identities', Uspekhi Mat. Nauk 20 (1965), 75-146.

[4] G. M. Bergman, 'Hyperidentities of groups and semigroups', Aequationes Math. 23 (1971), 50-65.

[5] J. Berman, 'Free spectra of 3-element algebras', Universal algebra and lattice theory, Proc. Puebla 1982, edited by R. S. Freese and O. O. Garcia, pp. 10-53, Lecture Notes in Math., vol. 1004, Springer-Verlag, Berlin and New York, 1983, pp. 10-53.

[6] T. Evans, 'Some connections between residual finiteness, finite embeddability and the word problem', J. London Math. Soc. (2) 1 (1969), 399-403.

[7] C. Fennemore, 'All varieties of bands', Semigroup Forum 1 (1970), 172-179. 
[8] J. A. Gerhard, 'The lattice of equational clases of idempotent semigroups', J. Algebra 15 (1970), 195-224.

[9] _ 'The number of polynomials of idempotent semigroups', J. Algebra 18 (1971), 366-376.

[10] _ , 'The word problem for semigroups satisfying $x^{3}=x$ ', Math. Proc. Cambridge Philos. Soc. 84 (1978), 11-19.

[11] J. A. Gerhard and M. Petrich, 'All varieties of regular orthogroups', Semigroup Forum 31 (1985), 311-351.

[12] _ - 'Word problem for free objects in certain varieties of completely regular semigroups', Pacific J. Math. 104 (1983), 351-359.

[13] G. Grätzer, Composition of functions, Proc. Conf. on Universal Algebra, pp. 1-106 (Queen's University, Kingston, Ont., 1969).

[14] G. Grätzer and R. Padmanabhan, 'On idempotent, commutative and nonassociative groupoids', Proc. Amer. Math. Soc. 28 (1971), 75-80.

[15] T. E. Hall and P. R. Jones, 'On the lattice of varieties of bands of groups', Pacific J. Math. 91 (1980), 327-337.

[16] A. Kisielewicz, 'Characterization of $p_{n}$-sequences for non-idempotent algebras', J. Algebra 108 (1987), 102-115.

[17] M. Petrich, Lectures in semigroups, (Wiley, London, 1977).

[18] W. Taylor, 'Hyperidentities and hypervarieties', Aequationes Math. 23 (1981), 30-49.

[19] W. Taylor, 'Equational logic', Houston J. Math. (1979).

Institute of Mathematics

Wroclaw University

Pl. Grunwaldzki 2/4

50-384 Wroclaw

Poland
Institute of Mathematics Technical University of Wroctaw Wybrzeze Wyspianskiego 27 50-370 Wroclaw

Poland 